Editor's Note: These short reviews of a recent paper in the Journal, written exclusively by graduate students or postdoctoral fellows, are intended to mimic the journal clubs that exist in your own departments or institutions. For more information on the format and purpose of the Journal Club, please see http://www.jneurosci.org/misc/ifa_features.shtml.

\title{
Speed Selectivity in V1: A Complex Affair
}

\author{
Bartlett D. Moore IV \\ Center for Neuroscience, University of California, Davis, California 95616 \\ Review of Priebe et al. (http://www.jneurosci.org/cgi/content/full/26/11/2941)
}

\section{Introduction}

Successful behavior in a visual environment requires knowing both what is being observed and where it is. To predict the future location of a moving object, the speed of the object must first be calculated. The middle temporal visual cortical area of the macaque (MT) is involved in visual motion processing, and speedselective neurons have been found there (Priebe et al., 2003). The primary visual cortex (V1) contributes to the directionselective responses of MT neurons (Movshon and Newsome, 1996), but does it also contribute to the speed tuning of neurons in MT?

Priebe et al. (2006) have investigated how and where in the visual system speed selectivity first arises (Fig. 1). Their recent article in The Journal of Neuroscience reports that a sizeable minority of complex cells in $\mathrm{V} 1$ possess robust speed selectivity, and the distribution of preferred speeds in these cells closely resembles that of neurons in MT. In contrast, V1 simple cells generally did not possess speed tuning that was independent of spatial frequency.

In arriving at these results, the authors used drifting sine wave gratings, a commonly used visual stimulus, to probe the speed selectivity of individual macaque V1 neurons. The structure of a drifting sine wave grating can be described using

Received May 2, 2006; revised May 23, 2006; accepted May 24, 2006. Correspondence should be addressed to Bartlett D. Moore, University of California, Davis-Center for Neuroscience, 1544 Newton Court, Davis, CA 95616. E-mail: bdmoore@ucdavis.edu.

DOI:10.1523/JNEUROSCI.1867-06.2006

Copyright $\odot$ 2006 Society for Neuroscience $\quad$ 0270-6474/06/267543-02\$15.00/0 only a few variables: contrast, spatial frequency, orientation, and temporal frequency. The drift speed is equivalent to the ratio of its temporal and spatial frequency, and a truly speed-tuned neuron will discharge vigorously to a particular drift speed regardless of the spatial or temporal frequencies used. If a neuron remains tuned to a particular temporal frequency regardless of the spatial frequency of the stimulus, it is described as being "separable" for spatiotemporal frequency. Such neurons are not speed selective.

When plotted in spatiotemporal frequency space, the response profiles of speed selective and separable neurons differ. Separable neurons have high responses to only one combination of spatial frequency and temporal frequency. Speed-selective neurons, however, will have high responses at more than one combination of spatial and temporal frequencies, and the response profile will be tilted along isospeed axes [Priebe et al. (2006), their Fig. 2 (http://www.jneurosci. org/cgi/content/full/26/11/2941/F2)].

Using a high-contrast stimulus and meticulous quantitative analysis, Priebe et al. (2006) found that approximately onequarter of direction-selective complex cells in V1 were tuned for a particular speed. The population distribution of speed-selective V1 complex cells was very similar to that of neurons in MT, in which approximately one-quarter are robustly speed tuned (Priebe et al., 2003). Simple cells, however, displayed essentially separable responses and as a population were not robustly speed selective [Priebe et al.
(2006), their Fig. 3 (http://www.jneurosci. org/cgi/content/full/26/11/2941/F3)].

The speed tuning of V1 complex cells, but not simple cells, depended on the contrast of the sine wave grating [Priebe et al. (2006), their Fig. 3 (http://www.jneurosci. org/cgi/content/full/26/11/2941/F3)]. Decreasing the contrast to $8 \%$ diminished speed selectivity and increased separability in most complex cells, again resembling the responses of MT cells (Priebe et al., 2003). Contrast gain was highest for spatial frequencies drifting at the same speed as that preferred in the low-contrast condition [Priebe et al. (2006), their Fig. 4 (http://www.jneurosci.org/cgi/content/ full/26/11/2941/F4)]. This finding suggests a possible mechanism for generating robust speed tuning at higher contrasts, and an avenue for future research.

The similarities in spatiotemporal response profiles of speed-tuned V1 and MT neurons support the hypothesis that speed tuning is generated first in V1 complex cells and is later inherited by MT cells through feedforward connections such as those described previously (Movshon and Newsome, 1996). Is there any additional evidence supporting the involvement of $\mathrm{V} 1$ in a speed-processing hierarchy?

To investigate this possibility, Priebe et al. (2006) presented more intricate stimuli composed of multiple overlapping sine wave gratings or drifting random dots. These two stimuli possess multiple spatial frequencies and are thus more similar to patterns encountered in natural environments. Moreover, the stimuli had been used previously to characterize MT neurons (Priebe et al., 2003) and thus were 
well suited to examine differences in motion processing between MT and V1.

Priebe et al. (2003) found that the preferred speed of MT neurons to grating stimuli consisting of multiple low spatial frequencies was poorly predicted by a linear sum of responses to individual gratings. V1 responses, however, were well predicted by a linear sum of the responses to single gratings [Priebe et al. (2006), their Fig. 6 (http://www.jneurosci.org/ cgi/content/full/26/11/2941/F6)]. In addition, MT neurons show increased speed selectivity to random dot stimuli (Priebe et al., 2003), but the speed tuning of V1 neurons remained constant [Priebe et al. (2006), their Fig. 7 (http://www.jneurosci. org/cgi/content/full/26/11/2941/F7)]. The authors suggest that the different response properties between $\mathrm{V} 1$ and MT may represent different stages of a motion-processing hierarchy progressing from the retina through V1 and into MT.

Overall, the results of Priebe et al. (2006) represent an important step in our understanding of how and where motion processing is accomplished in the visual system. Although the authors dealt more with documenting the emergence of speed selectivity than with the mechanisms through which it arises, they did nonetheless propose a role for contrast gain in reducing separability in V1 complex cells. This intriguing proposal certainly warrants additional experimental and theoretical elucidation.

Along these lines, the direction selectivities of some neurons in cat area 17 have been reported to vary with temporal frequency (Saul and Humphrey, 1992) and

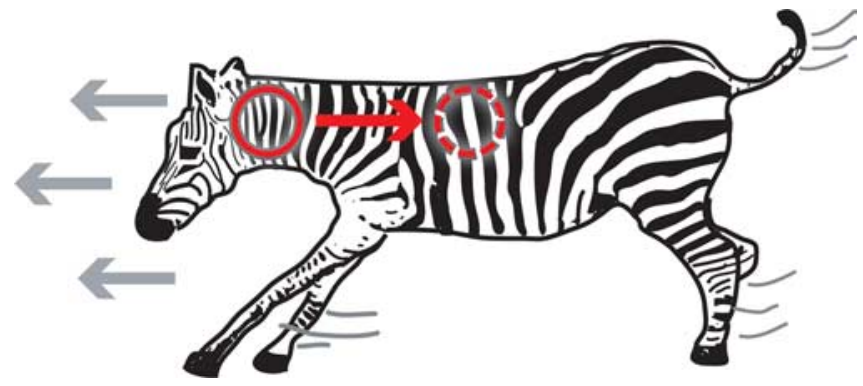

Figure 1. Schematic diagram illustrating the presentation of various spatiotemporal frequencies to the receptive field of a V1 neuron. The portion of the surface of a moving object presented to a stationary receptive field (solid red ring) will occupy positions sliding along the axis of motion of the object (red arrow). In this example, a neuron tuned to specific spatial and temporal frequencies will be unable to encode the zebra's speed because these properties change as the zebra moves across the receptive field. In contrast, a speed-selective neuron would be mostly unaffected by the changing spatial and temporal frequencies and would have its discharge rate entrained by the zebra's speed. Priebe et al. (2006) provide evidence for the existence of speedselective neurons among the complex cells in primary visual cortex but not among simple cells.

contrast (Peterson et al., 2006). It remains to be determined whether speed-tuned macaque V1 neurons display these inconsistencies. If they do, this could have important implications for theories of speed tuning in visual cortex

Perhaps the most salient message that Priebe et al. (2006) deliver is that V1 is an integral part of a speed-processing hierarchy that includes MT. Although this may indeed prove to be true, one must also consider other sources of input to MT. MT receives feedforward input from a variety of structures, including the pulvinar, V2, V3, and the LGN (Sincich et al., 2004). Indeed, V1 input to MT appears to be quite limited, albeit highly stereotyped (Movshon and Newsome, 1996). Moreover, MT and V1 are targets of feedback connections from a variety of brain structures. Thus it remains possible that V1 and MT neurons arrive at their respective speed selectivities through similar but independent circuits.

\section{References}

Movshon JA, Newsome WT (1996) Visual response properties of striate cortical neurons projecting to area $\mathrm{MT}$ in macaque monkeys. J Neurosci 16:7733-7741.

Peterson MR, Li B, Freeman RD (2006) Direction selectivity of neurons in the striate cortex increases as stimulus contrast is decreased. J Neurophysiol 95:2705-2712.

Priebe NJ, Cassanello CR, Lisberger SG (2003) The neural representation of speed in macaque area MT/V5. J Neurosci 23:5650-5661.

Priebe NJ, Lisberger SG, Movshon JA (2006) Tuning for spatiotemporal frequency and speed in directionally selective neurons of macaque striate cortex. J Neurosci 26:2941-2950.

Saul AB, Humphrey AL (1992) Temporalfrequency tuning of direction selectivity in cat visual cortex. Vis Neurosci 8:365-372.

Sincich LC, Park KF, Wohlgemuth MJ, Horton JC (2004) Bypassing V1: a direct geniculate input to area MT. Nat Neurosci 7:1123-1128. 